\title{
Study on the gas sensitivity of vanadium-doped molybdenum disulfide to mustard gas
}

\author{
Huaizhang Wang ${ }^{1}$, Yangyang Wang ${ }^{1}$, Pengbo $\mathrm{Chi}^{1,2}$, Huaning Jiang ${ }^{1,3}$, Jingfei Chen ${ }^{1,4}$, Ting \\ $\mathrm{Li}^{5}$, and Ting Liang ${ }^{1, *}$ \\ ${ }^{1}$ The Institute of NBC Defense, Chinese PLA Army, Beijing, China \\ ${ }^{2}$ No. 32296 Troops of the Chinese PLA, Shenyang, Liaoning, China \\ ${ }^{3}$ No. 32281 Troops of the Chinese PLA, Chengdu, Sichuan, China \\ ${ }^{4}$ No. 32169 Troops of the Chinese PLA, Linzhi, Xizang Autonomous Region, China \\ ${ }^{5}$ No. 61699 Troops of the Chinese PLA, Zhijiang, Hubei, China
}

\begin{abstract}
As a graphene-like material, molybdenum disulfide has similar properties to graphene, but due to its excellent properties such as adjustable band gap, molybdenum disulfide has a broader application in many aspects (such as gas sensors). With the deepening of research, molybdenum disulfide cannot fully meet the needs of researchers due to defects and other reasons. Therefore, researches on doping and compounding of molybdenum disulfide have gradually attracted attention. At present, most of the research on gas sensitivity has focused on harmful gases (such as nitrogen dioxide, ammonia and carbon monoxide, etc.). There are few studies on the erosive chemical toxic mustard gas. In this paper, vanadiumdoped molybdenum disulfide were prepared based on chemical vapor deposition, and the gas-sensitive response of vanadium-doped molybdenum disulfide to mustard gas was studied.
\end{abstract}

Keywords: Molybdenum disulfide, vanadium doping, mustard gas, gas sensitivity.

\section{Introduction}

Mustard gas was one of the main chemical warfare agents developed and used during the First World War, and there are still large stocks in some countries[1,2]. Pure mustard gas is a transparent, colorless liquid with almost no odor. Some impurities give the mustard gas a greasy appearance and the typical smell of garlic. Mustard gas is almost insoluble in water, but has good solubility in alcohol and organic solvents. Its freezing point is $14.4^{\circ} \mathrm{C}$ and its boiling point is between 215 and $217^{\circ} \mathrm{C}[3]$. Mustard gas is a foaming agent that can cause skin blisters, respiratory tract damage, eye disease, and bone marrow suppression. Despite a lot of research work in the past 90 years, no specific mustard gas antidote has been discovered[4].

In recent years, there have been reports of terrorist attacks and major public emergencies around the world. Our country has a large population and densely populated

\footnotetext{
*Corresponding author: 1525093476@qq.com
} 
areas, and its ability to defend against chemical terrorist attacks is relatively weak. Once a terrorist attack occurs, social stability and economic development will be severely damaged. In addition, incidents of mustard gas abandoned in China by the Japanese invaders have repeatedly occurred, causing serious physical, mental and spiritual damage to the Chinese people. Therefore, rapid and accurate detection of trace amounts of mustard gas is an urgent question.

Chemical agent sensor has become an important means of chemical agent analysis and detection. There are many types of sensing principles, including ion mobility spectroscopy, flame photometry, semiconductors, etc. Among them, the resistive semiconductor gas sensor has attracted much attention because of its excellent sensing performance, simple preparation process and low cost. Among them, the resistive semiconductor gas sensor has attracted much attention because of its excellent sensing performance, simple preparation process and low cost. The sensitivity of the sensor depends on the adsorption between the surface of the material and the gas molecules.

In 2004, Novoselov and Geim of the University of Manchester in the United Kingdom successfully peeled off single-layer graphene using micromechanical peeling method[5]. Since then, two-dimensional materials have become a hot spot in the field of materials science due to their extremely large specific surface area. With the continuous deepening of materials science research, new graphene-like materials transition metal chalcogenides have gradually entered the field of vision of researchers. Transition metal sulfides also show their unique physical and chemical properties. The compound has great application prospects in many fields such as optoelectronic devices, catalysis, energy conversion, storage and sensing [6-10]. All the constituent atoms of a single-layer transition metal sulfide are exposed to the surrounding environment. Its large specific surface area provides good conditions for the adsorption of gas molecules on the surface. The sheet layer can be used as an electron donor or acceptor. The transfer between the two-dimensional sheet and the adsorbed gas molecules can change the resistance and carrier density of the single-layer transition metal sulfide. This is the principle of its application in gas sensors[11,12].

Among transition metal sulfides, molybdenum disulfide $\left(\mathrm{MoS}_{2}\right)$ is the earliest and most widely studied material [13-14]. A large number of previous experiments have shown that $\mathrm{MoS}_{2}$ is an ideal transition metal sulfide that can be used as a gas-sensing material for chemical sensors[15-17]. However, when intrinsic $\mathrm{MoS}_{2}$ is exposed to the air, its surface easily adsorbs oxygen, resulting in a significant decrease in its carrier concentration and carrier mobility, resulting in unsatisfactory gas sensitivity[18]. Therefore, the intrinsic $\mathrm{MoS}_{2}$ gas sensor device needs to work in a vacuum or inert atmosphere to maximize its excellent gas sensitivity characteristics, which severely limits the application of the intrinsic $\mathrm{MoS}_{2}$ gas sensor device in military field poison detection. Doping the intrinsic molybdenum disulfide with other metal atoms can make up for its original defects, effectively reduce the combined ratio of the material and the oxygen in the gas to be measured, and significantly improve the sensing performance[19,20]. This article intends to dope $\mathrm{V}$ element into $\mathrm{MoS}_{2}$ to solve the problems of low response and poor stability of twodimensional MoS2 in the air, and initially explore the response of V-doped molybdenum disulfide $\left(\mathrm{V}-\mathrm{MoS}_{2}\right)$ to mustard gas.

\section{Experimental section}

\subsection{Material preparation}

The gas-sensitive materials in this study are all prepared by chemical vapor deposition. Pure $\mathrm{MoS}_{2}$ is obtained by vaporizing molybdenum trioxide powder and sulfur powder at 
high temperature and finally depositing on the substrate. The preparation method of the two-dimensional $\mathrm{MoS}_{2}$ material doped with $\mathrm{V}$ is similar to the above process. But the difference is that in the preparation process of the doping material, a certain amount of $\mathrm{VO}_{2}$, $\mathrm{MoO}_{3}$ and $\mathrm{KCl}$ must be thoroughly ground and mixed in a mortar to form a uniformly mixed solid of niobium oxide and molybdenum oxide as the metal source for growth. The addition of $\mathrm{KCl}$ is to increase the volatilization rate of the metal source into the gas phase. The temperature of the sulfur sublimation zone is $180^{\circ} \mathrm{C}$, the temperature of the deposition growth zone is $650^{\circ} \mathrm{C}$, and the growth pressure is $2000 \mathrm{~Pa}$. The deposition time is $15 \mathrm{~min}$ to obtain the V-doped $\mathrm{MoS}_{2}$ material. The schematic diagram of the process is shown in Figure 1.

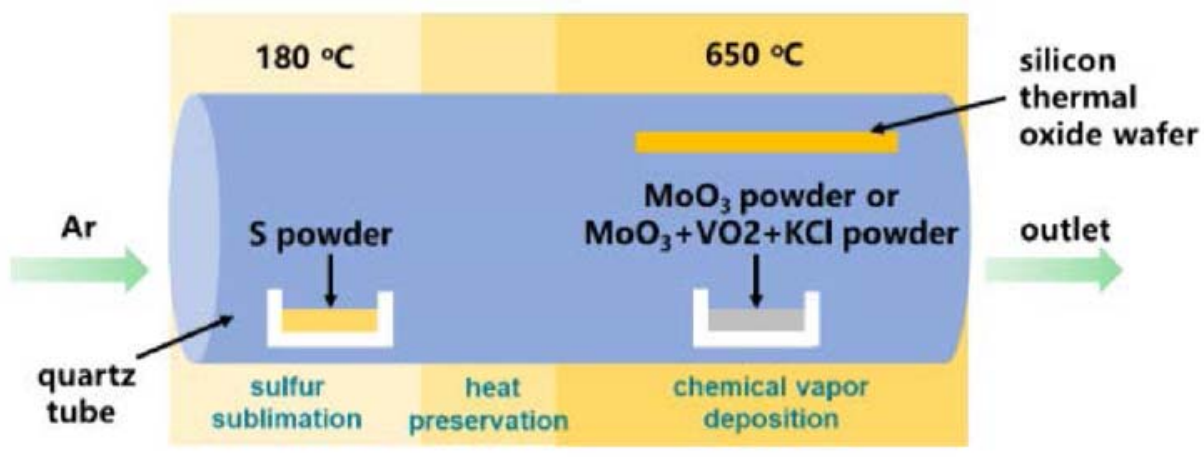

Figure 1. Schematic diagram of preparation of $\mathrm{V}-\mathrm{MoS}_{2}$ by chemical vapor depositio

\subsection{Characterizations}

The most intuitive characterization methods of two-dimensional layered materials are various microscopes, including scanning electron microscopy, atomic force microscopy, etc. In this paper, the morphology of the samples was characterized by scanning electron microscopy (SEM), and the thickness of the films was identified by atomic force microscopy (AFM) and Raman spectroscopy (Raman). The relative content of each element in the sample was determined by EDS. X-ray photoelectron spectroscopy (XPS) is an important surface analysis method, which can determine the composition and chemical valence state of the surface elements through the electron binding energy. All XPS spectra were corrected by C $1 \mathrm{~s}(284.8 \mathrm{EV})$.

\subsection{Fabrication of gas sensor}

In order to facilitate the application of electrical signals to the $\mathrm{MoS}_{2}$ thin-film gas sensor and accurately measure its surface resistance, it is necessary to pre-make the lead electrodes on the silicon substrate. Firstly, a mask of interdigital shape is pasted on the surface of the cleaned and dried substrate. The interdigital electrode mask used in the experiment is made of $0.1 \mathrm{~mm}$ thick alloy sheet by laser. Then, the substrate covered with the mask was put into the vacuum chamber of the magnetron sputtering equipment. The metal gold was used as the target and argon as the working gas. Under the conditions of argon flow rate of 25 $\mathrm{ml} / \mathrm{min}$, substrate temperature of $25^{\circ} \mathrm{C}$, background vacuum less than $4 \times 10^{-4} \mathrm{~Pa}$ and sputtering pressure of $2.0 \mathrm{~Pa}$, platinum interdigital electrode with thickness of $0.1-0.3 \mu \mathrm{m}$ was obtained. 


\subsection{The occurrence of dynamic air currents}

Mustard gas (purity more than 98\%) is provided by The Institute of NBC Defense. The method is as follows: a certain amount of liquid agent is injected into the volatilization chamber, the orifice plate is covered and the upper cover is tightened, and placed in the poison generator. Adjust the cold trap to keep the agent in the liquid state in the generator. Through the gas mass flow controller, the constant air flow is introduced, and the poison vapor diffused from the volatilization chamber is carried out, and the stable poisoned gas flow is formed after dilution. In the process of the experiment, the temperature of cold trap, the pore size in the center of volatile orifice plate and the flow rate and ratio of purge and dilution gas flow can be adjusted to produce dynamic agent gas flow with different concentrations.

\subsection{Gas sensing measurements}

Connect the gas circuit, power line and data transmission line of the detection system, turn on the sensor power supply and the upper computer control software, realize the signal connection, first connect the dry clean air into the detection room, and make it interact with the two-dimensional $\mathrm{MoS}_{2}$ sensitive film on the sensor array. After the signal generated is counted by the resistance value acquisition device, it is sent to the computer for recording by the recording software, and the air sensor is obtained The working curve of the parts in the clean air flow. After the baseline is stable, the air will be switched to the gas flow with known concentration of mustard gas, and then switch to air after a certain time. When the resistance value of load is restored to the initial value or near the initial value, the toxic air flow shall be injected again, and the above process shall be repeated several times to ensure that the sensitivity fluctuation of repeated test of response sensitivity under the same test condition is less than $10 \%$. Use the self-built intelligent gas sensitivity analysis system to test the gas sensitivity performance and study the sensitivity of the gas sensor to the mustard gas detection $\mathrm{S}$ :

$$
\mathrm{S}(\%)=100 * \frac{\Delta \mathrm{R}}{\mathrm{R}_{0}}=100 * \frac{\mathrm{K}_{\mathrm{g}}-\mathrm{K}_{0}}{\mathrm{R}_{0}}
$$

Among them, $R_{0}$ and $R_{g}$ are the resistance values of the thin film gas sensor in the air and the gas flow of the sample to be tested.

\section{Results and discussion}

\subsection{Structure and morphology}

\subsubsection{Scanning electron microscope}

Figure 2 shows the SEM image of V-MoS 2 . It can be seen that the V-doped samples have the needle like morphology. 


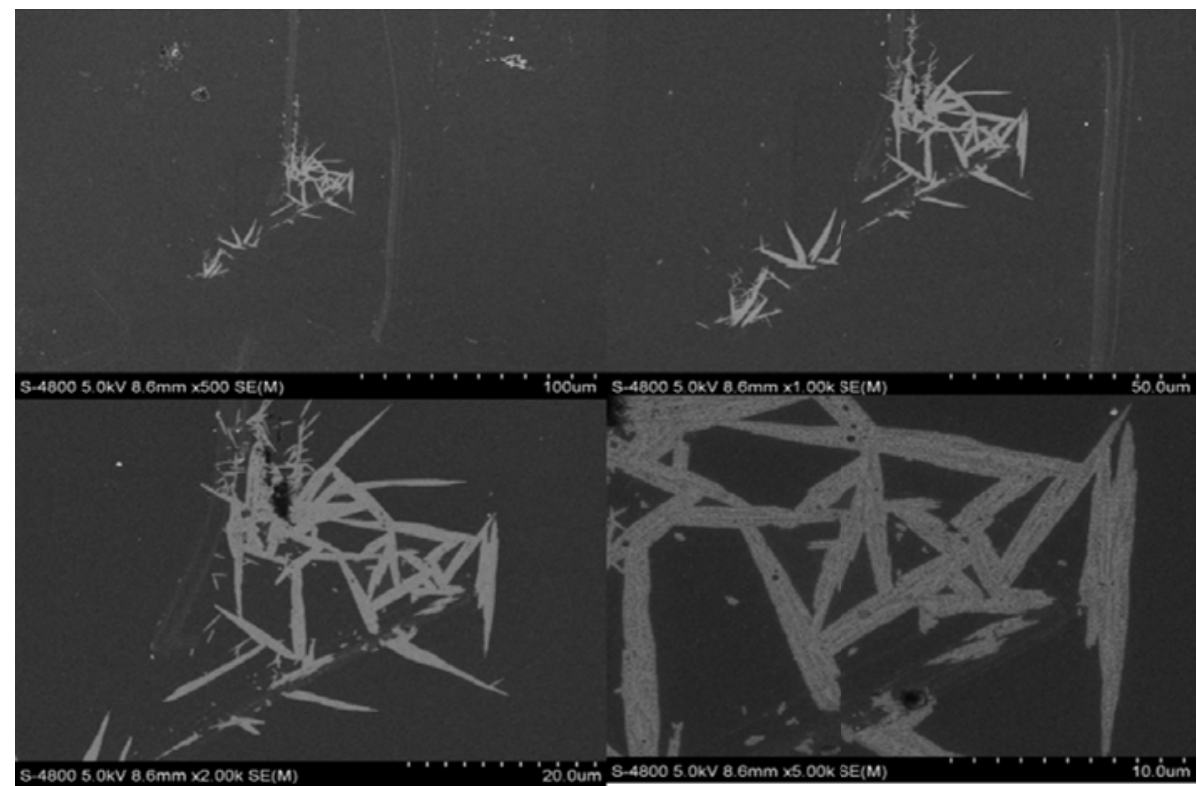

Figure 2. SEM of V-MoS 2

\subsubsection{Atomic force microscope}

As shown in Figure 3 is the height profile of $\mathrm{V}-\mathrm{MoS}_{2}$ with few layers of $\mathrm{MoS}_{2}$. It can be seen that the thickness of $\mathrm{V}-\mathrm{MoS}_{2}$ sample sheet is about $3.8 \mathrm{~nm}$, which is consistent with the thickness of 4-5 layer $\mathrm{MoS}_{2}$ material.

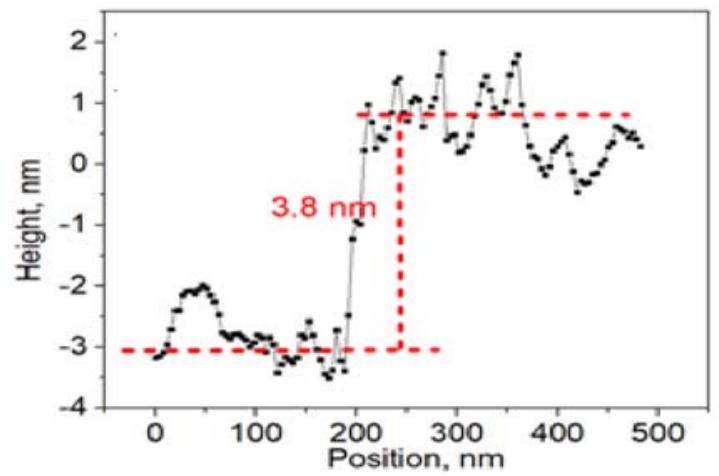

Figure 3. Quantitative height profile corresponding to AFM images of $\mathrm{V}-\mathrm{MoS}_{2}$ sample

\subsubsection{Raman}

The Raman spectra of $\mathrm{MoS}_{2}$ before and after $\mathrm{V}$ doping are shown in Figure 4. It can be seen from the figure that the introduction of $\mathrm{V}$ has little effect on the position of the inplane vibration peak $E_{2 g}^{1}$ and the interlayer vibration characteristic peak $A_{1 g}$ of $\mathrm{S}$ atom, and the wave number difference between the two characteristic peaks has no obvious change, which indicates that the addition of a small amount of $\mathrm{V}$ has little effect on the structure of $\mathrm{MoS}_{2}$ film. In the same way, it can be seen from the Raman spectra that the 
intensity ratio of the in-plane vibration peak $E_{2 g}^{1}$ of $\mathrm{S}$ atom to that of the interlayer vibration peak $A_{1 g}$ of $\mathrm{S}$ atom decreases after introducing a small amount of $\mathrm{V}$.

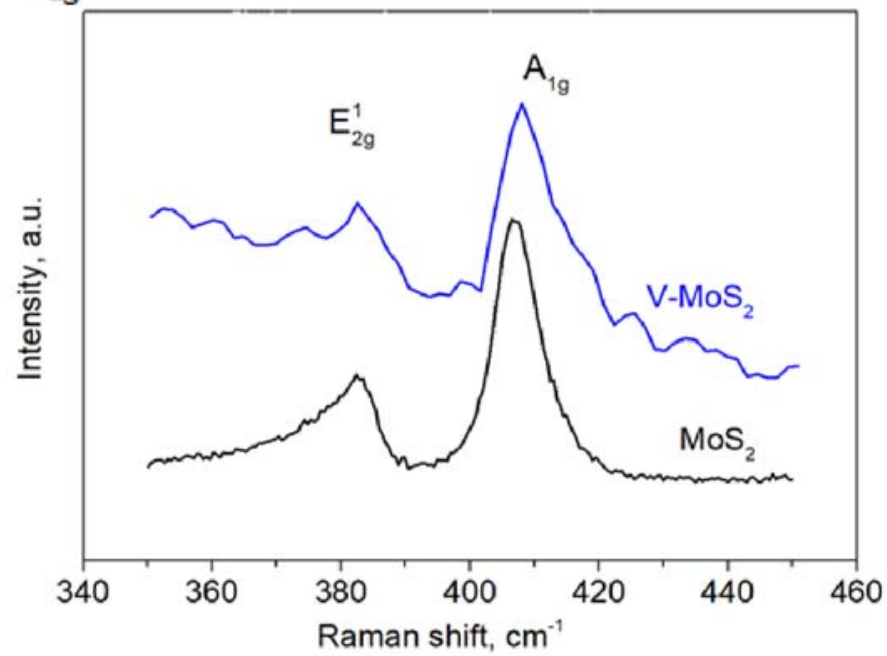

Figure 4. Raman of $\mathrm{MoS}_{2}$ and $\mathrm{V}-\mathrm{MoS}_{2}$ samples

\subsubsection{Energy Dispersive X-Ray Spectroscopy (EDS)}

EDS is a method to determine the relative content of elements in the sample by analyzing the characteristic X-ray wavelength and intensity of the sample. Figure 5 is the SEM-EDS mapping diagram of $\mathrm{V}-\mathrm{MoS}_{2}$ sample. It can be seen that the distribution of Mo, S and $\mathrm{V}$ in the sample is highly uniform, which also confirms that $\mathrm{V}$ element can be well doped into two-dimensional $\mathrm{MoS}_{2}$ by chemical vapor deposition.

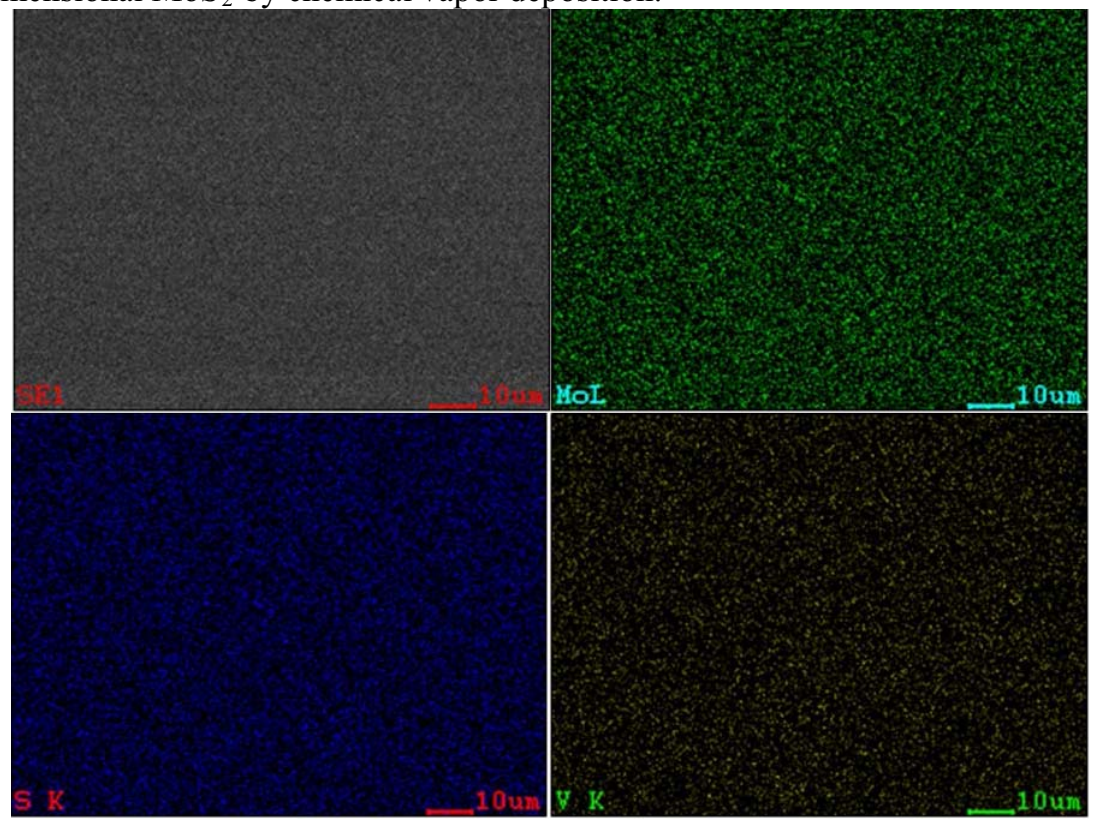

Figure 5. SEM-EDS mapping diagram of $\mathrm{V}-\mathrm{MoS}_{2}$ 


\subsubsection{X-ray photoelectron spectroscopy (XPS).}

Figure 6(a) shows the full XPS spectrum of $\mathrm{V}-\mathrm{MoS}_{2}$ sample. It can be seen that the characteristic peaks of the main elements in the samples are very obvious, and are consistent with the XPS spectra of $\mathrm{MoS}_{2}$. Figure 6(b) shows the high resolution scanning spectrum of $\mathrm{V}$ in the narrow region of $2 \mathrm{p}$ orbit in $\mathrm{V}-\mathrm{MoS}_{2}$ sample. It can be seen that there are characteristic peaks of $\mathrm{VS}_{2}$ at $514.36 \mathrm{eV}$ and $521.86 \mathrm{eV}$ in this region. Based on the above XPS results, it is shown that V-MoS 2 materials can be successfully synthesized by vapor deposition method.

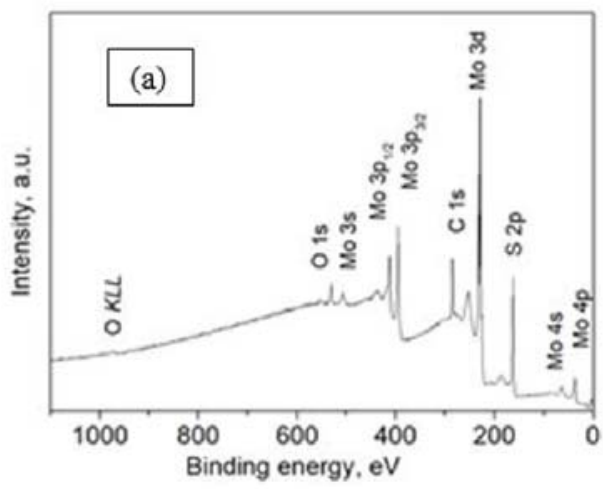

(a) XPS spectrum

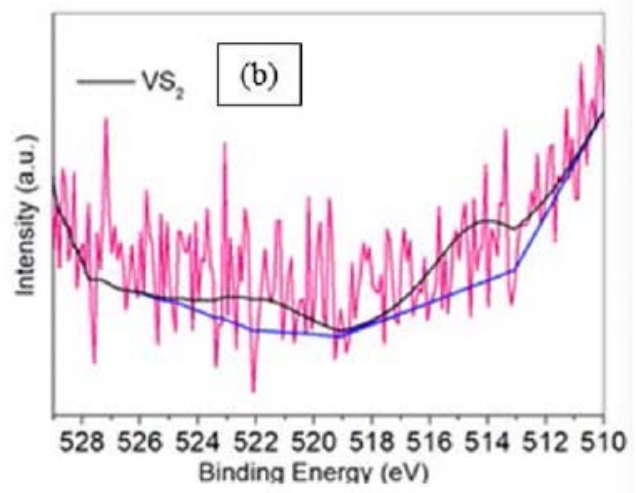

(b) Fitting results of $V 2 p$ characteristic peak

Figure 6. X-ray photoelectron spectroscopy of $\mathrm{V}-\mathrm{MoS}_{2}$

\subsection{Gas sensing properties}

\subsubsection{Response of different concentrations of mustard gas}

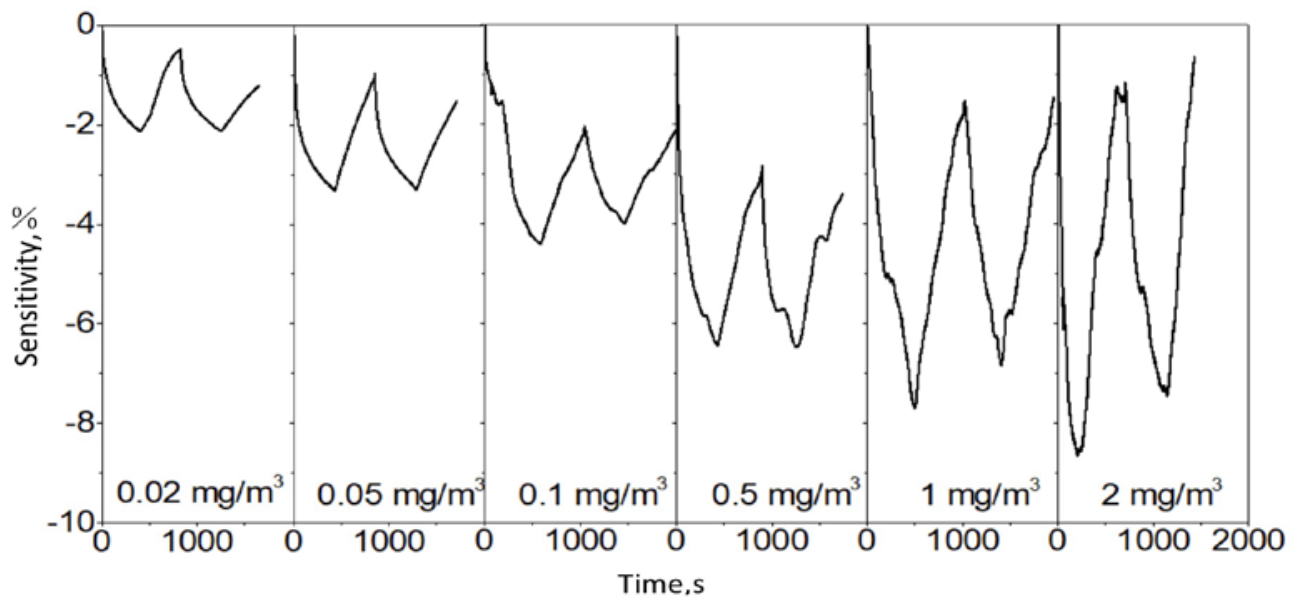

Figure 7. Comparison of response curves of $\mathrm{V}-\mathrm{MoS}_{2}$ in different concentrations of mustard gas

The response curves of $\mathrm{V}-\mathrm{MoS}_{2}$ samples to different concentrations of mustard gas were obtained by gradually reducing the concentration of mustard gas in the contaminated gas stream. The results are shown in Figure 7. It can be seen that in different concentrations of mustard atmosphere, with the extension of response time, the response sensitivity of the materials first decreased rapidly and then tended to be flat. When the concentration of 
mustard gas is low, the response curve is very smooth, while when the concentration of mustard gas is high, the response curve appears noise, which may be due to the uneven local gas mixing in the high concentration mustard gas experiment. In view of the actual needs of the detection of toxic agents, we focused on the response performance of sensitive materials at low concentrations. When the concentration of mustard gas was $0.02 \mathrm{mg} / \mathrm{m}^{3}$ (2.8 ppb), the response curve was still clear, and the response degree was $2.13 \%$ under the experimental conditions.

\subsubsection{Repeatability of response to mustard gas}

In order to test the repeatability of response of $\mathrm{V}-\mathrm{MoS}_{2}$ sensitive material, mustard gas at the same concentration was continuously measured for 5 times. The results show that V$\mathrm{MoS}_{2}$ has good repeatability for 5 times of mustard gas detection of 0.1 and $2 \mathrm{mg} / \mathrm{m}^{3}$, and the sensitivity fluctuation is less than $10 \%$ after deducting the baseline fluctuation factor, which indicates that this kind of material has good repeatability detection.

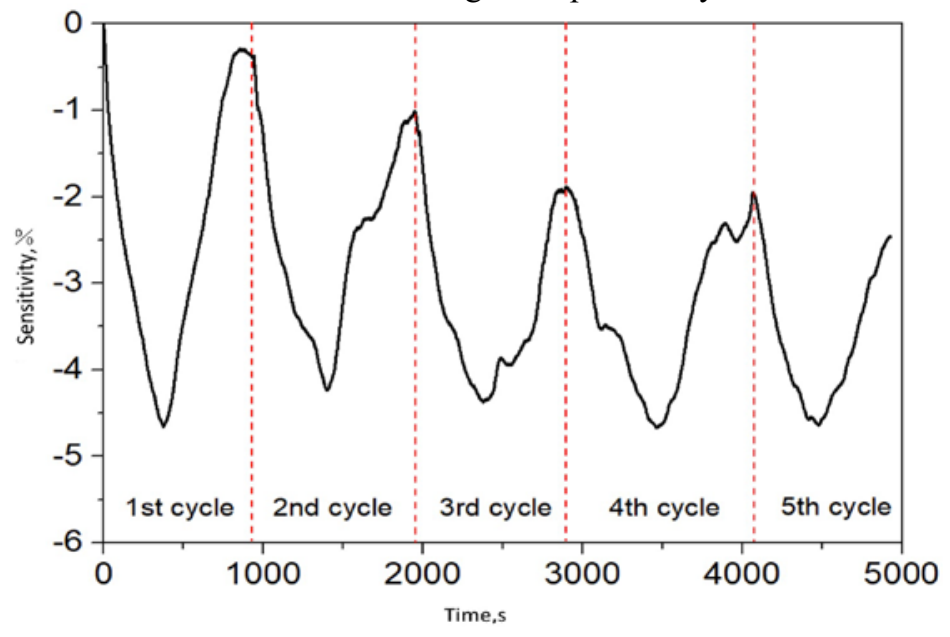

Figure 8. Repeatability of response of $\mathrm{V}-\mathrm{MoS}_{2}$ to $0.1 \mathrm{mg} / \mathrm{m}^{3}$ mustard gas

\subsubsection{Stability of response to mustard gas}

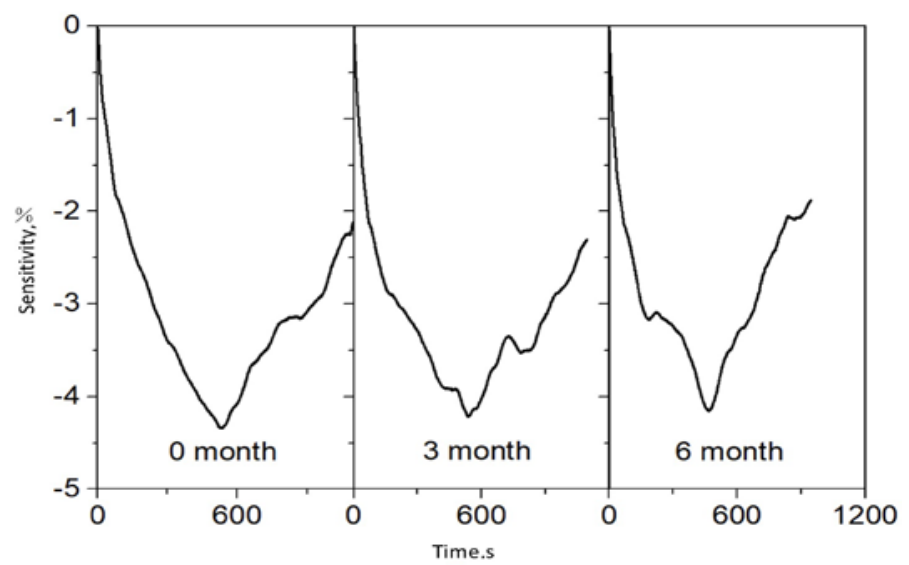

Figure 9. Response stability of $\mathrm{V}-\mathrm{MoS}_{2}$ to $0.1 \mathrm{mg} / \mathrm{m}^{3}$ mustard gas 
After the sensor is placed for a period of time, its response performance to $0.1 \mathrm{mg} / \mathrm{m}^{3}$ mustard gas is tested, and the results are shown in Figure 9. It can be seen from the figure that the performance of mustard gas detection has no obvious change after the sensor is placed for 3 months and 6 months, and the change of response amplitude SM is less than $10 \%$, which indicates that this kind of sensitive material has good stability in air.

\section{Conclusions}

In this paper, a few-layer V-doped two-dimensional MoS2 was successfully prepared by chemical vapor deposition, and a sensor for the erosive agent mustard gas was developed using it as a sensitive material. The experimental results show that the $\mathrm{V}-\mathrm{MoS}_{2}$ sample has a high response to mustard gas, and shows good repeatability and stability in mustard gas detection. In this experiment, the detection limit of the two-dimensional $\mathrm{V}-\mathrm{MoS}_{2}$ low-layer material for mustard gas is as low as $0.02 \mathrm{mg} / \mathrm{m}^{3}$, which can meet the requirements for onsite identification and detection of mustard gas at trace levels. This indicates that $\mathrm{V}$-doped molybdenum disulfide is expected to be a gas-sensitive material for the mustard gas sensor of erosive agent.

\section{Acknowledgments}

This work was financially supported by Military research fund (zhuangzong[2018]635).

\section{References}

1. Wang QQ, Begum RA, Day VW, Bowman-James K, Sulfur, oxygen, and nitrogen mustards: stability and reactivity, J. Org Biomol Chem. 10 (2012)8786-8793.

2. Reiter $\mathrm{G}$, Mueller S, K oller M, Thiermann H, WorekBundeswehr F, In vitro toxicokinetic studies of cyclosarin: Molecular mechanisms of elimination, J. Toxicol Lett. 227(2014)1-11.

3. Borak J, Sidell FR, Agents of chemical warfare: sulfur mustard, J. Ann Emerg Med. 21(1992)303-308.

4. Jan YH, Heck DE, Malaviya R, et al, Cross-linking of thioredox-in reductase by the sulfur mustard analogue mechlorethamine (methylbis (2-chloroethyl)amine)in human lung epithelial cells and rat lung: selective inhibition of disulfide reduction but not redox cycling, J . Chem Res Toxicol, 27 (2014) 61-75.

5. Novoselov, K.S., Geim, A.K., Morozov, S.V., et al, Electric field effect in atomically thin carbon films, J. Science. 306(2004) 666-669.

6. Jin W, Kai T, Miao J, et al, Graphene for Future High-Performance Gas Sensing, J . 2017.

7. Choi S J, Kim I D, Recent Developments in 2D Nanomaterials for ChemiresistiveType Gas Sensors, J. Electronic Materials Letters, 14(2018):1-40.

8. Li Q, Lu J, Gupta P, et al, Engineering Optical Absorption in Graphene and Other 2D Materials: Advances and Applications, J. Advanced Optical Materials, 7(2019).

9. Donarelli, Maurizio;Ottaviano, Luca. 2D Materials for Gas Sensing Applications: A Review on Graphene Oxide, $\mathrm{MoS}_{2}, \mathrm{WS}_{2}$ and Phosphorene, J. SENSORS, 14(2018)145

10. Perkins F K, Friedman A L, Cobas E, et al, Chemical Vapor Sensing with Monolayer $\mathrm{MoS}_{2}$, J. Nano letters, 13(2013) 668-673.

11. Yue Q, Shao Z, Chang S, et al, Adsorption of Gas Molecules on Monolayer $\mathrm{MoS}_{2}$ and Effect of Applied Electric Field , J . Nanoscale research letters, 8(2013)425. 
12. Tongay S, Zhou J, Ataca C, et al, Broad-range Modulation of Light Emission in Twodimensional Demiconductors by Molecular Physisorption Gating , J . Nano letters, 13(2013) 2831-2836.

13. Choi W., Choudhary N., Han G.H., et al, Recent development of two-dimensional transition metal dichalcogenides and their applications, J. Mater. Today, 20(2017)116-130.

14. Zappa, D, Molybdenum Dichalcogenides for Environmental Chemical Sensing, J. Materials, 10(2017)1418.

15. Kumar R, Goel N, Kumar M, High performance $\mathrm{NO}_{2}$ sensor using $\mathrm{MoS}_{2}$ nanowires network, J. Applied Physics Letters, 5(2018)1.

16. Sharma S, Kumar A, Kaur D, AIP Room temperature ammonia gas sensing properties of $\mathrm{MoS}_{2}$ nanostructured thin film, J.1(2018).

17. Burman D, Ghosh R, Santra S, et al, Role of vacancy sites and UV-ozone treatment on few Layered $\mathrm{MoS}_{2}$ Nanoflakes for Toxic Gas Detection, J. Nanotechnology, 2017.

18. Barsan N, Weimar U, Conduction Model of Metal Oxide Gas Sensors, J. Journal of Electroceramics, 7(2001)143-167

19. G Zhao, M Li, Ni-doped $\mathrm{MoS}_{2}$ biosensor: a promising candidate for early diagnosis of lung cancer by exhaled breathe analysis, J. Applied Physics A: Materials Science \& Processing, 124(2018).

20. J Zhu, H Zhang, Y Tong, L Zhao, et al, First-principles investigations of metal (V, Nb, Ta)-doped monolayer $\mathrm{MoS}_{2}$ : Structural stability, electronic properties and adsorption of gas molecules, J. Applied Surface Science, 419(2017) 